\title{
ON THE ZEROS OF DIRICHLET SERIES ASSOCIATED WITH CERTAIN CUSP FORMS ${ }^{1}$
}

\author{
JAMES L. HAFNER
}

As is well known, in 1859 Riemann [6] conjectured that the function $\zeta(s)$ defined in $\operatorname{Re} s>1$ by the Dirichlet series $\sum_{n=1}^{\infty} n^{-s}$ has all its zeros, apart from the "trivial" zeros at the negative even integers, on the line $\operatorname{Re} s=\frac{1}{2}$. It is known that these "nontrivial" zeros lie symmetrically about the line Re $s=$ $\frac{1}{2}$ within the strip $0<\operatorname{Re} s<1$. The truth of this Riemann Hypothesis would have a profound impact in the theory of numbers, particularly with regard to the distribution of primes.

One of the major achievements in this theory was due to Selberg [7] in 1943. He proved for $s(s)$ that a positive proportion of the nontrivial zeros lie on the critical line. Later authors have given specific numerical values for this proportion. In this note we announce the proof of a similar theorem for Dirichlet series attached to certain cusp forms on the full modular group. We formulate the specific theorem below.

Let $F(z)$ be a holomorphic cusp form of even integral weight $k$ and constant multiplier system for the full modular group $\Gamma(1)=S L(2, \mathbf{Z}) /\{ \pm I\}$. That is,

$$
F(M z)=(c z+d)^{k} F(z), \quad M=\left(\begin{array}{cc}
* & * \\
c & d
\end{array}\right) \in \Gamma(1),
$$

and $F(z)$ vanishes at $i \infty$. Expand $F(z)$ in a "Fourier series" at the cusp $i \infty$ as

$$
F(z)=\sum_{l=1}^{\infty} f(l) e^{2 \pi i l z}
$$

The Dirichlet series $L_{f}(s)=\sum_{l=1}^{\infty} f(l) l^{-s}$ converges absolutely for

$$
\operatorname{Re} s>(k+1) / 2
$$

and can be continued to an entire function in the $s$-plane. Furthermore, $L_{f}(s)$ has all its nontrivial zeros in the strip $(k-1) / 2<\operatorname{Re} s<(k+1) / 2$. Let

$$
N(T)=\#\left\{\rho=\beta+i \gamma: 0<\gamma<T,(k-1) / 2<\beta<(k+1) / 2, L_{f}(\rho)=0\right\}
$$

and

$$
N_{0}(T)=\#\left\{\rho=k / 2+i \gamma: 0<\gamma<T, L_{f}(\rho)=0\right\} .
$$

It is known [4] that $N(T) \sim c T \log T$ for some constant $c>0$. We then have the following theorem.

Received by the editors July 14, 1982 and, in revised form, September 7, 1982.

1980 Mathematics Subject Classification. Primary 10D24, 10H10, $10 \mathrm{D} 12$.

${ }^{1}$ Research partially supported by NSF Grant MCS 77-18723A03 at the Institute for Advanced Study.

(C) 1983 American Mathematical Society 
THEOREM. If $F(z)$ is an eigenfunction of all the Hecke operators with $f(1)=1$, then there exists a constant $A>0$ depending on $F$ such that

$$
N_{0}(T)>A T \log T
$$

for all sufficiently large $T$.

The assumptions on $F$ insure that (a) $f(l)$ is a multiplicative function, (b) $|f(p)| \leq 2 p^{(k-1) / 2}, p$ a prime, and (c) $f(l)$ is real for all $l$, in fact, a totally real algebraic number. Fact (b) is due to Deligne [1] and (c) can be found in Ogg [5, p. II-11 and p. III-11]. Of course $f(1)=1$ can always be achieved by the appropriate normalization.

The method of proof uses three main ingredients. First, we modify slightly Selberg's idea to introduce a mollifier $\phi(s)$ which approximates $L_{f}^{-1 / 2}$. Secondly, we require an approximate functional equation for $L_{f}(s)$ which is provided by A. Good [2]. Finally we need to extend A. Good's techniques [3] for computing

$$
\int_{0}^{T}\left|L_{f}\left(\frac{k}{2}+i t\right)\right|^{2} d t \sim c T \log T .
$$

We are then required to estimate expressions like

$$
\int_{0}^{T}\left|L_{f}\left(\frac{k}{2}+i t\right)\right|^{2}\left|\phi\left(\frac{k}{2}+i t\right)\right|^{4} d t
$$

There are some extra difficulties which we encounter which make this theorem quite difficult. First, the coefficients $f(l)$ are not completely multiplicative. This makes certain arithmetical sums more difficult to analyse. Secondly, as is the difficulty in (1), analysis of the series

$$
\sum_{l=1}^{\infty} \frac{f(l) f(l+n)}{(l+n / 2)^{s}}, \quad n \geq 0,
$$

is required. This is obtained by appealing to the spectral theory of the Laplacian acting on $L^{2}(\Gamma(1) \backslash \mathcal{K})$. (See Good [3].) However the introduction of the mollifier complicates significantly the corresponding calculation in (2). In particular we need an analysis of the series

$$
\sum_{l=1}^{\infty} \frac{f(l) f((l b+n) / a)}{(l b+n / 2)^{s}}, \quad n \geq 0,(a, b)=1 .
$$

We require growth estimates with respect to the $\operatorname{Im} s$, in the region where the series does not converge absolutely, and which are uniform in $a$ and $b$. This uniformity is the major difficulty. To handle this problem we appeal to spectral theory of $L^{2}\left(\Gamma_{0}(a, b) \backslash \not\right)$ where $\Gamma_{0}(a, b)$ is the congruence group defined by

$$
\Gamma_{0}(a, b)=\left\{\left(\begin{array}{cc}
* & \beta \\
\gamma & *
\end{array}\right) \in \Gamma(1): \beta \equiv 0(\bmod b), \gamma \equiv 0(\bmod a)\right\}
$$

And finally, estimates for the Fourier coefficients of the Maass wave forms (the orthonormal basis of eigenfunctions for the discrete spectrum of the Laplacian) which are uniform in $a$ and $b$ are required. 
The mollifier we choose is given by

$$
\phi(s)=\phi_{\xi}(s)=\sum_{\nu \leq \xi} \alpha_{\nu}\left(1-\frac{\log \nu}{\log \xi}\right)
$$

where $\xi \geq 2$ and $\alpha_{l}=\mu(l) f(l) / d(l)$. Here $\mu$ and $d$ are the usual Möbius and divisor functions. We then prove that there exists a number $a>0$ such that for $\xi=T^{a}, 0 \leq h \leq(\log \xi)^{-1 / 2}$,

$$
\left.\left.\int_{0}^{T}\left|\int_{t}^{t+h} L_{f}\left(\frac{k}{2}+i u\right)\right| \phi\left(\frac{k}{2}+i u\right)\right|^{2} d u\right|^{2} d t \ll \frac{T h^{3 / 2}}{\sqrt{\log \xi}}
$$

and

$$
\int_{0}^{T} \int_{t}^{t+h}\left|L_{f}\left(\frac{k}{2}+i u\right) \phi^{2}\left(\frac{k}{2}+i u\right)\right|^{2} d u d t \ll \frac{h^{2} T \log T}{\log \xi} .
$$

From these estimates the deduction of the theorem follows just as in Selberg's proof.

A classical example to which our theorem applies is the cusp form of weight 12 defined by

$$
\begin{aligned}
\Delta(z) & =q \prod_{n=1}^{\infty}\left(1-q^{n}\right)^{24}, \quad q=e^{2 \pi i z} \\
& =\sum_{l=1}^{\infty} \tau(l) e^{2 \pi i l z}
\end{aligned}
$$

where $\tau(l)$ is Ramanujan's function.

\section{REFERENCES}

1. P. Deligne, La conjecture de Weil. I, Inst. Hautes Études Sci. Publ. Math. 43 (1974), 273-307.

2. A. Good, Approximative Funktionalgleichungen und Mittelwertsätze für Dirichletreihen, die Spitzenformen assoziiert sind, Comment. Math. Helv. 50 (1975), 327-361.

3. __, Beitraege zur Theorie der Dirichletreihen, die Spitzenformen zugeordnet sind, J. Number Theory 13 (1981), 18-65.

4. C. G. Lekkerkerker, On the zeros of a class of Dirichlet series, Dissertation, Utrecht, 1955.

5. A. Ogg, Modular forms and Dirichlet series, Benjamin, New York, 1969.

6. B. Riemann, Ueber die Anzahl der Primzahlen unter einer gegebenen Grösse, Gesammelte Werke, Teubner, Leipzig, 1892; reprinted by Dover, New York, 1953.

7. A. Selberg, On the zeros of Riemann's zeta-function, Skr. Norske. Vid.-Akad. Oslo I 10 (1942), 59 pp.

Department of Mathematics, California institute of Technology, PASADENA, CALIFORNIA 91125 